\title{
Efficacy of Hilbert-Huang Transform (HHT) in the Analysis of Instantaneous Low Frequency Waves of Magnetosheath
}

\author{
Ekong U. Nathaniel, Nyakno J. George*, Jewel I. Ibanga, Aniekan M. Ekanem \\ Department of Physics, Akwa Ibom State University, Ikot Akpaden, Nigeria \\ Email: "nyaknojimmyg@gmail.com, "nyaknogeorge@aksu.edu.ng
}

Received 23 November 2015; accepted 10 January 2016; published 13 January 2016

Copyright (C) 2016 by authors and Scientific Research Publishing Inc.

This work is licensed under the Creative Commons Attribution International License (CC BY). http://creativecommons.org/licenses/by/4.0/

(c) (i) Open Access

\section{Abstract}

The flow of supersonic plasma is accompanied by a highly thermalized region called the Magnetoshealth found after the bow shock. Enclosed within this region are different wave modes associated with classes of boundaries which have been determined by different methods. The efficacy of Hilbert-Huang transform (HHT) is based on the conditionality of allowing for the local analysis of frequencies, which presents the physical meaning of the original signal at that instant. The observed data have been taken from Cluster II Fluxgate Magnetometer (FGM) instrument that provides advantage for the analysis in three dimensions. The result compares favourably with instantaneous frequencies computed using simple Hilbert transform (SHT) with electric field measurements of Cluster II mission already carried out in literatures. The result of this study has shown that HHT provides the best applicability in the magnetosheath data analysis than the wavelet and Fast Fourier Transform (FFT). The application of HHT based on its advantages over other methods is viewed to be very critical in the analysis of multi-frequency signals where different frequencies could be determined distinctively at a point.

\section{Keywords}

Plasma Waves, Instantaneous Frequency, Empirical Mode Decomposition (EMD), Hilbert-Huang Transform (HHT)

\section{Introduction}

The flow of supersonic plasma is accompanied by a highly thermalized region called the Magnetoshealth found

${ }^{*}$ Corresponding author.

How to cite this paper: Nathaniel, E.U., George, N.J., Ibanga, J.I. and Ekanem, A.M. (2016) Efficacy of Hilbert-Huang Transform (HHT) in the Analysis of Instantaneous Low Frequency Waves of Magnetosheath. International Journal of Geosciences, 7, 11-19. http://dx.doi.org/10.4236/ijg.2016.71002 
after the bow shock [1]. Hilbert-Huang Transform (HHT) according to Huang [2] considers the combination of empirical mode decomposition technique and the simple Hilbert transformation. The first part of this combination involves the extraction of different oscillatory modes (known as intrinsic mode functions, IMFs), which constitutes the distorted data waveform [3] [4]. This principally centres on the extraction (sifting) of IMFs $\left(C_{i}\right)$ from a real valued data $S(t)$ by subtracting the enveloped mean of the maxima and minima formed using cubic spline interpolation. As a matter of fact, $C_{1}$ carries the shortest periodic component of the signal. Their mean is considered as the average of the upper and lower lines, and the real valued signal data $S(t)$ is given in general form as expressed in Equation (1)

$$
S(t)=\sum_{i=1}^{i} C_{i}(t)+R_{i}
$$

where $R_{i}$ denotes the residue from the difference of $S(t)$ and the IMF.

\subsection{Hilbert Spectrum (HT)}

The next part of HHT is the Hilbert spectrum which is the plot of the instantaneous frequency of the signal versus time [5]. The real data is composed of many different oscillatory modes of IMFs. The $C_{i}(t)$ components, the Hilbert transform is given for a real valued signal $S(t)$ as according to [4] as

$$
H\left(C_{i}(t)\right)=\frac{\xi}{\pi} \int_{-\infty}^{+\infty} \frac{C_{i}(\tau)}{t-\tau} \mathrm{d} \tau
$$

where $H$ is the Hilbert transform operator and $\xi$ signifies the Cauchy principal value of the integral.The IMFs are suitably analyzed using the Hilbert transform. The IMFs analytical signal complex conjugate pair was constructed according to [6] in the expression given in Equation (3) as:

$$
\begin{gathered}
Z_{i}(t)=X_{i}(t)+j Y_{i}(t) \\
X_{i}(t)+j Y_{i}(t)=a_{i}(t) \exp \left(j \theta_{i}(t)\right)
\end{gathered}
$$

where $j H_{i}(X(t))$ is the Hilbert filter defined as The signals $Y_{i}(t)$ and $X_{i}(t)$, form the complex conjugates defining the analytic signal $Z_{i}(t)$ with the instantaneous wave amplitude magnitude of $a_{i}(t)$ expressed as

$$
a_{i}(t)=\sqrt{X_{i}^{2}(t)+Y_{i}^{2}(t)}
$$

The instantaneous phase angle $\theta$ is computed using Equation (6)

$$
\theta(t)=\arctan \left(\frac{Y(t)}{X(t)}\right)
$$

The instantaneous frequency $\omega(t)$ according to [7]-[10] is defined as $\frac{\mathrm{d} \theta(t)}{\mathrm{d} t}$ for each IMF. Using the Hilbert transform to analyze the frequency profile of the signal means that we do not encounter the time-frequency uncertainty associated with Fourier-based transforms.

\subsection{Basis of HHT}

IMFs are defined as functions with zero mean and having as many zero crossings as maxima or minima. IMFs are 'mono-component' and application of simple Hilbert transform leads to determination of instantaneous frequency. HHT is suitable for non-linear and non-stationary data exhibiting distortion in waveform with fast changing frequencies such as space plasma and fields. Data are adaptively decomposed into different oscillatory components (called Intrinsic Mode Functions (IMFs)) of different time scales that are intrinsic to the data [11].

\section{Observations and Results}

The data used in this work were taken from cluster data archive. The magnetic field and electric field data were those observed by cluster instruments. The magnetic field data used in the analysis were recorded by fluxgate 
magnetometer [12] and taken from the magnetosheath, a region of highly fluctuated and thermalized plasma [13]-[15]. Ten minutes data between 020000 and 021000 UT were taken from Cluster C1, C2, C3 and C4 on 01 01, 2001. The profiles are given in Figure 1 and Figure 2. Figure 1 and Figure 2 represent the magnetic field in nanotesla (nT) and electric field in millivolt per metre $(\mathrm{mV} / \mathrm{m})$. They both have similar waveform which serves as the basis for comparison of the magnetic field data used in this work with the electric field data used by Carozzi [16]. Figure 3 and Figure 4 show the plots resulting from the application of EMD. The intrinsic mode functions (IMFs) for y-component of magnetic and electric fields data (C1) on 01 01, 2001 from 020000 to 021000 UT are displayed. From the top to the bottom, panels show original data, 1st, 2nd, 3rd and 4th IMFs. The last is the residue which is not an IMF. The horizontal axis is the time in seconds. Figure 5 shows the Hilbert spectra of all the IMFs (1 - 4) of data of y component on C1. The energy of the spectra is mainly under the nyquist frequency of $0.12 \mathrm{~Hz}$. Figure 6 shows the spectrogram of all the IMFs. The vertical dash lines indicate the average frequencies of each of the IMFs. This shows that the first IMF has a frequency above nyquist

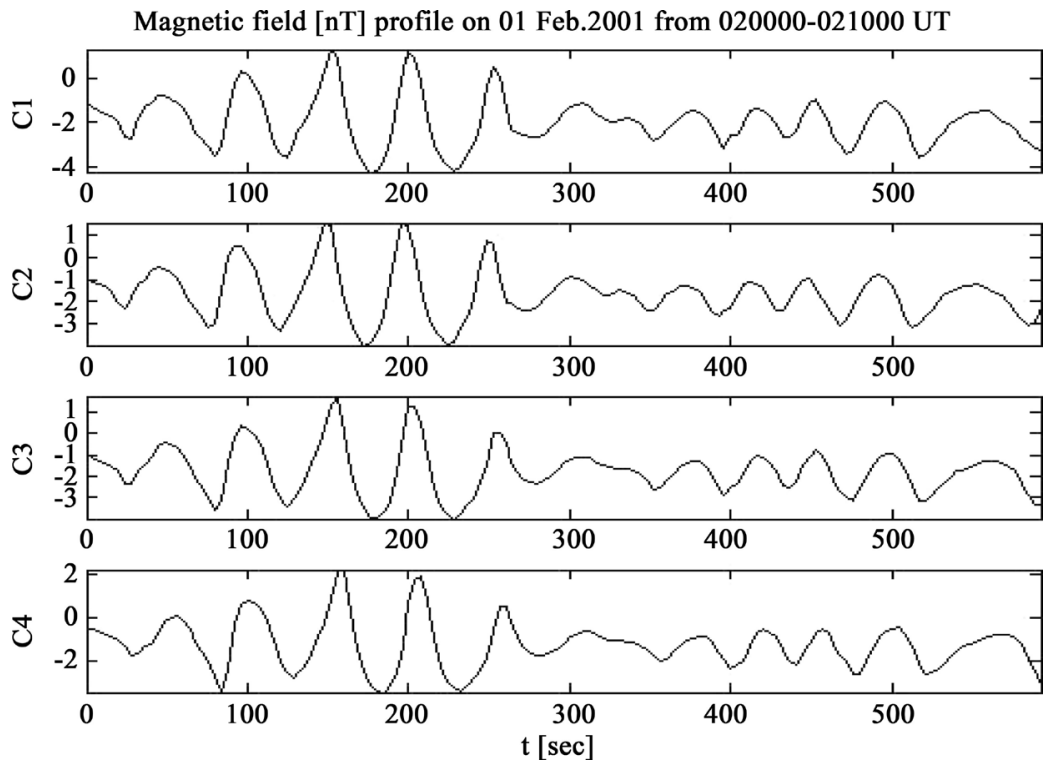

Figure 1. Magnetic field profile for the original signal.

Electric field [mW/M] profile on 01 Feb.2001 from 020000-021000 UT
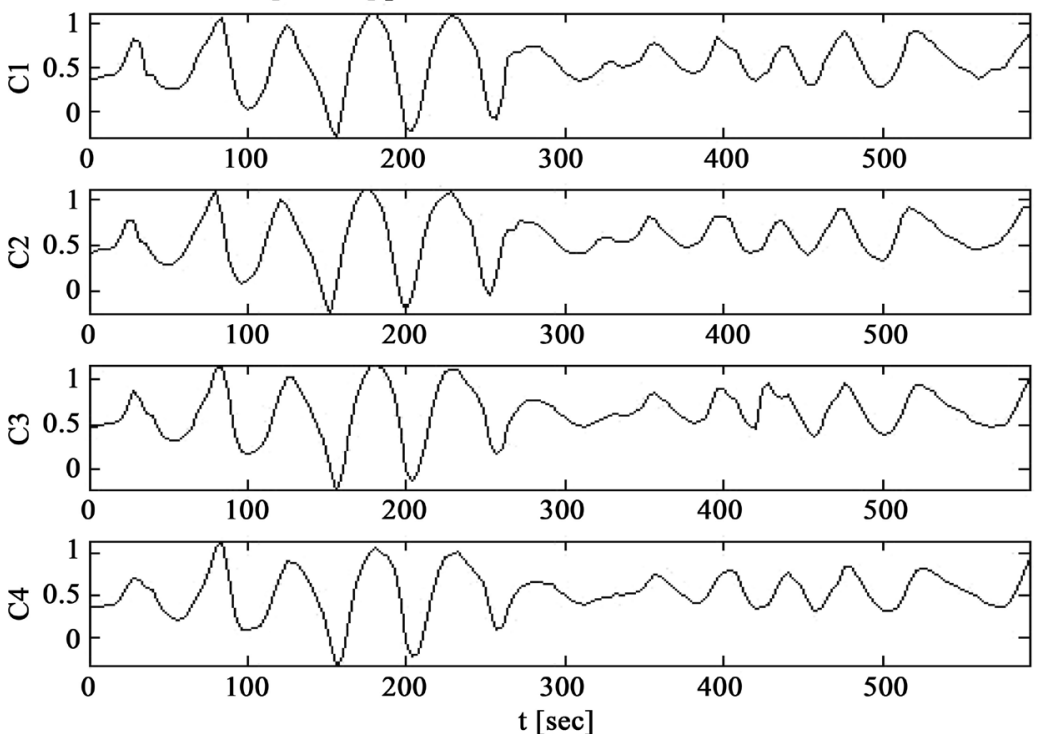

Figure 2. Electric field profile for the original signal. 

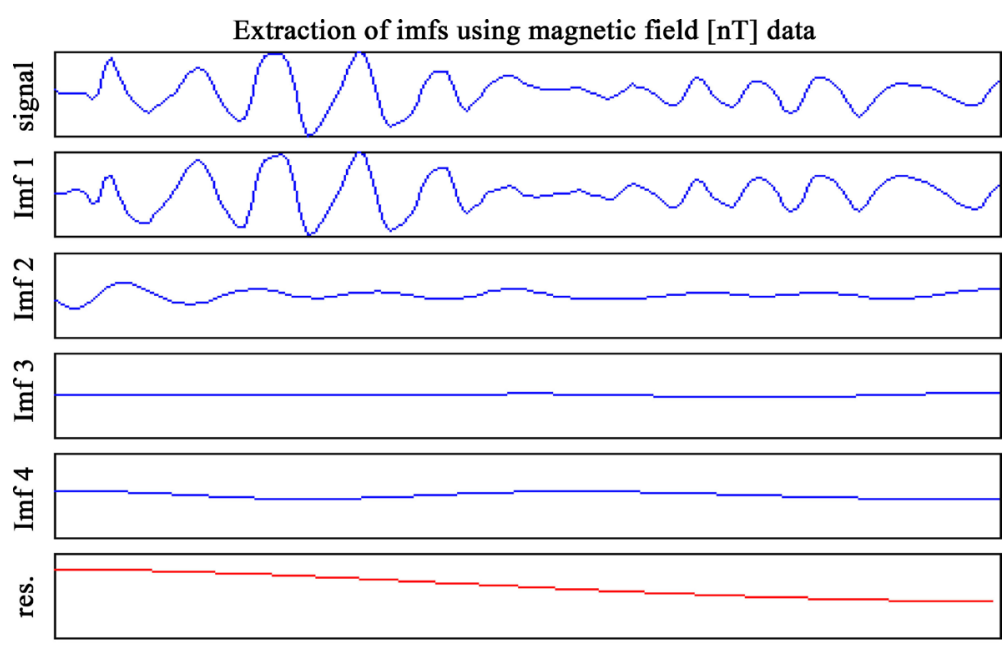

$\mathbf{t}[\mathbf{s}]$

Figure 3. EMD of magnetic field.
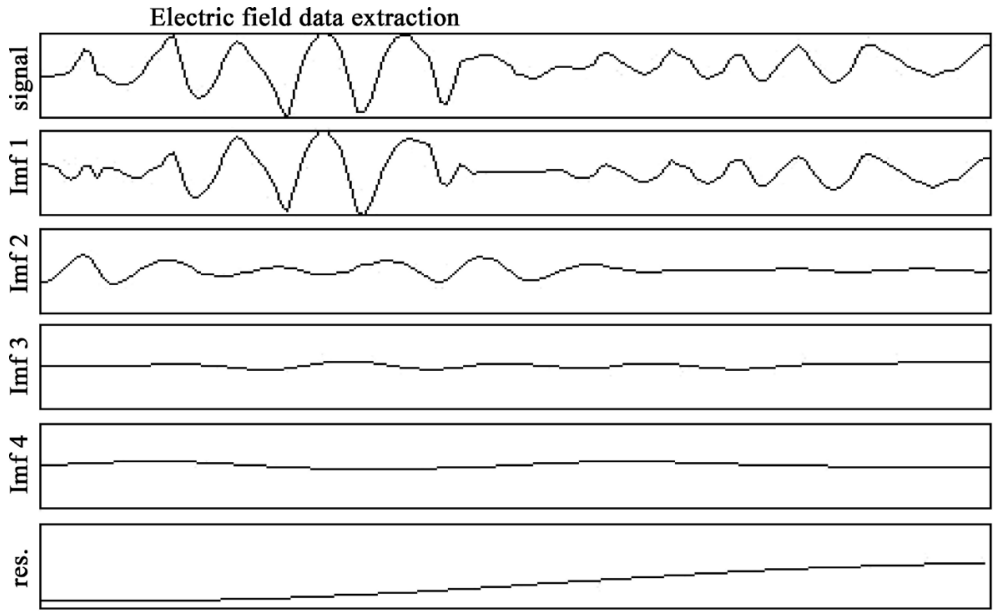

t [s]

Figure 4. EMD of electric field.

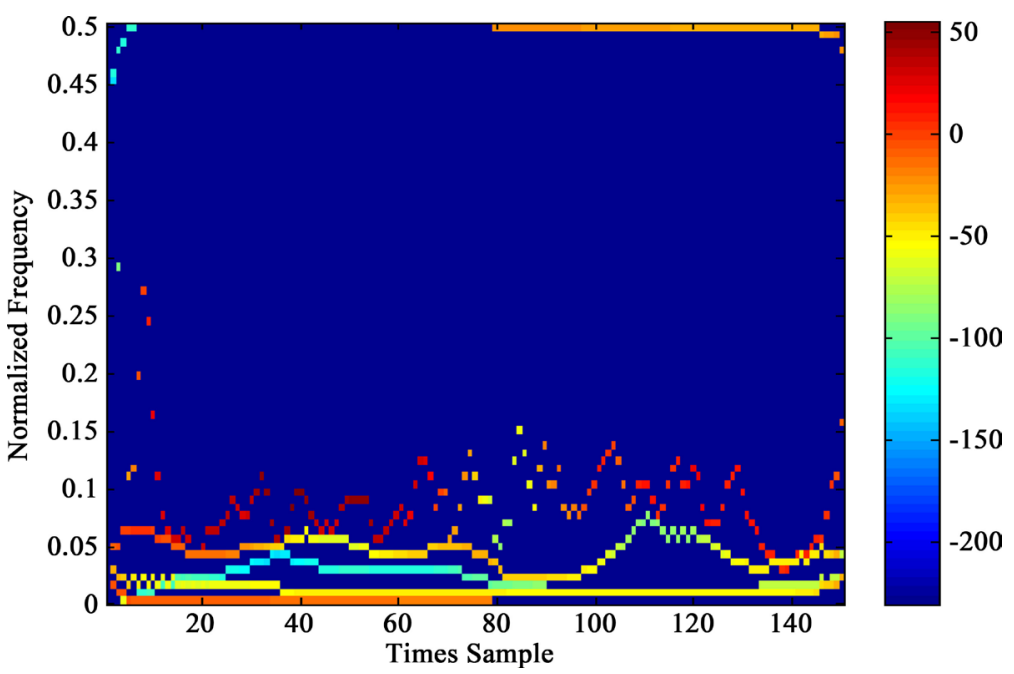

Figure 5. Hilbert spectra of the IMFs obtained from HHT. 
and is thus discarded. Figure 7 represents a scalogram of the first IMF with scales of 6 corresponding to 0.0208 $\mathrm{Hz}$. This is about the value of frequency of $0.025 \mathrm{~Hz}$ when compared to the original data. Figure 8 gives a scalogram with a scale 9 corresponding to $0.0139 \mathrm{~Hz}$. Figure 9 is a representation of scalogram of the third IMF with scale of 37 corresponding to $0.005 \mathrm{~Hz}$. Figure 10 equally represents the scalogram of the fourth IMFs with a scale of 45 corresponding to a frequency of $0.005 \mathrm{~Hz}$. Figure 11 displays the instantaneous frequencies of the two (2nd and 3rd) IMFs that meet the conditions of the information carrier. We used the ratio of energy character rate [3] of the original data and that of the individual IMFs to set a threshold that distinguishes noise from the information carrier.

Between 0 and 80 seconds on Figure 1 and Figure 2 corresponding to 0 and 20 sample points on Figure 11, there is an anti-phase superposition pattern which actually leads to the destructive interference. This is an indication of signal superposition rather than having a signal.

Between 80 and 320 seconds on Figure 1 and Figure 2 corresponding to 20 and 80 sample points, there is phase superposition pattern. The in-phase superposition leads to the constructive interference of signals which

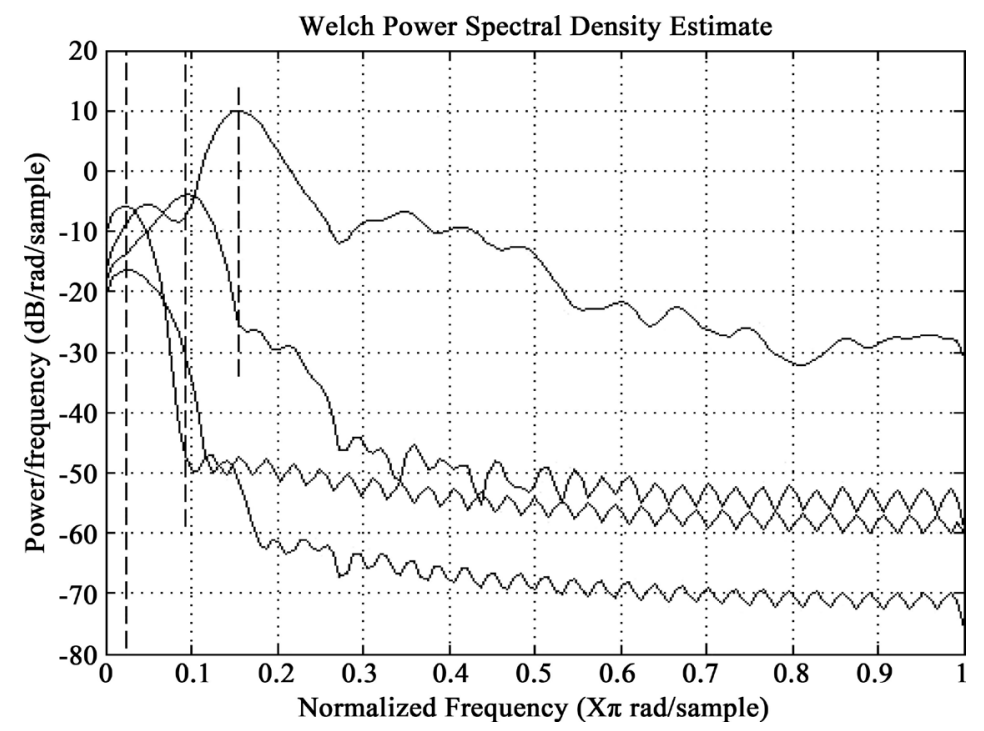

Figure 6. Spectrogram of the IMFs obtained from Fourier transform.
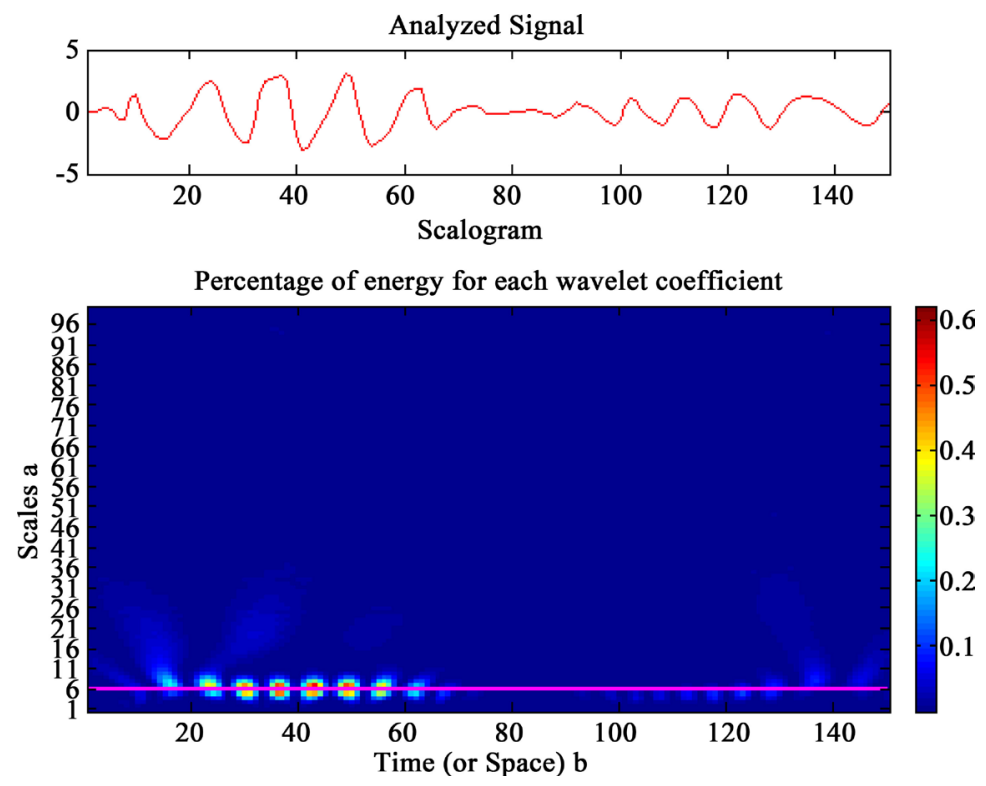

Figure 7. Scalogram of first IMF obtained from wavelet transform. 


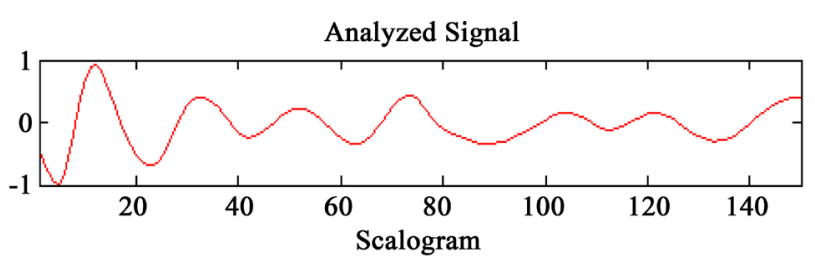

Percentage of energy for each wavelet coefficient

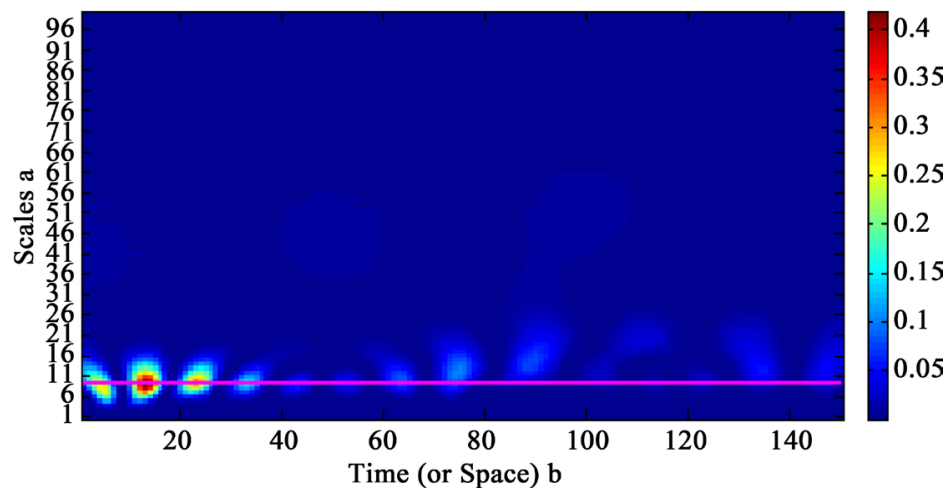

Figure 8. Scalogram of second IMF obtained from wavelet transform.
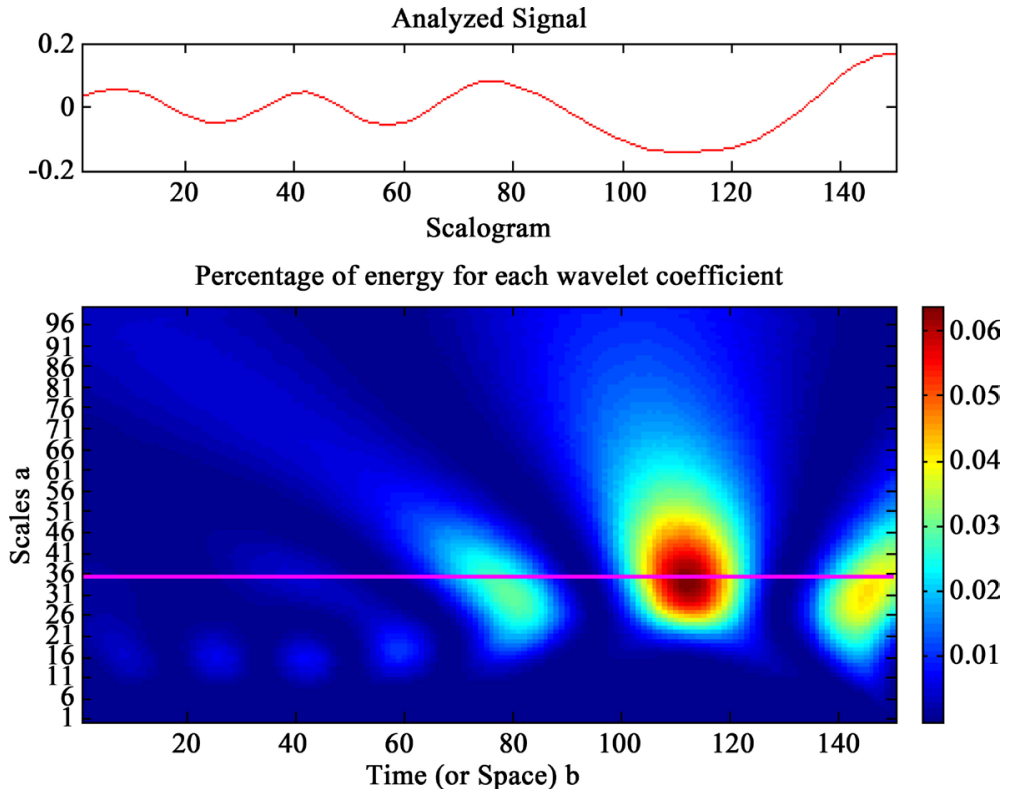

Figure 9. Scalogram of third IMF obtained from wavelet transform.

enhances the resulting signal amplitudes. Again, between 320 and 600 seconds, there is a recovery of anti-phase superposition in Figure 1 and Figure 2. The result obtained with electric field data collected within the same period gives instantaneous frequency profile similar to the wave with lower instantaneous frequency on Figure 11.

Table 1 displays the comparisons of the different methods used in the signal analysis. From Table 1, HHT is found to be adaptive or usable in different conditions while Fourier and wavelet transforms are non-adaptive as they cannot be adjusted for use in different conditions. The reason for this assertion is due to the ability of the HHT to have frequency at any instant or point considered. This suggests the reason for considering the HHT as having frequencies that are differentially local while the convolved frequencies in Fourier and wavelet transforms are respectively global and regional. Inability of the wavelet and Fourier transforms to display instantaneous frequencies make them to be considered as non-adaptive in signal processing. In terms of variable pres- 
entation, Hilbert-Huang transform (HHT) and wavelet transform can on analysis give information on Energy, time and frequency while Fourier transform can only give information on Energy and frequency. The HHT is non-linear while wavelet and Fourier are all linear. While HHT and wavelet transform are non-stationary, Fourier transform is stationary. The result of this study has shown that (HHT) provides the best applicability in the magnetosheath data analysis than the wavelet and Fast Fourier Transforms which instead of providing information at instant, respectively give the needed information on regional and global scales. The application of HHT would be very useful in the analysis of multi-frequency signal where different frequencies could be determined distinctively at point.

\section{Conclusion}

Using HHT with particular attention to the improvements on EMD shows a low frequency wave and other fre-
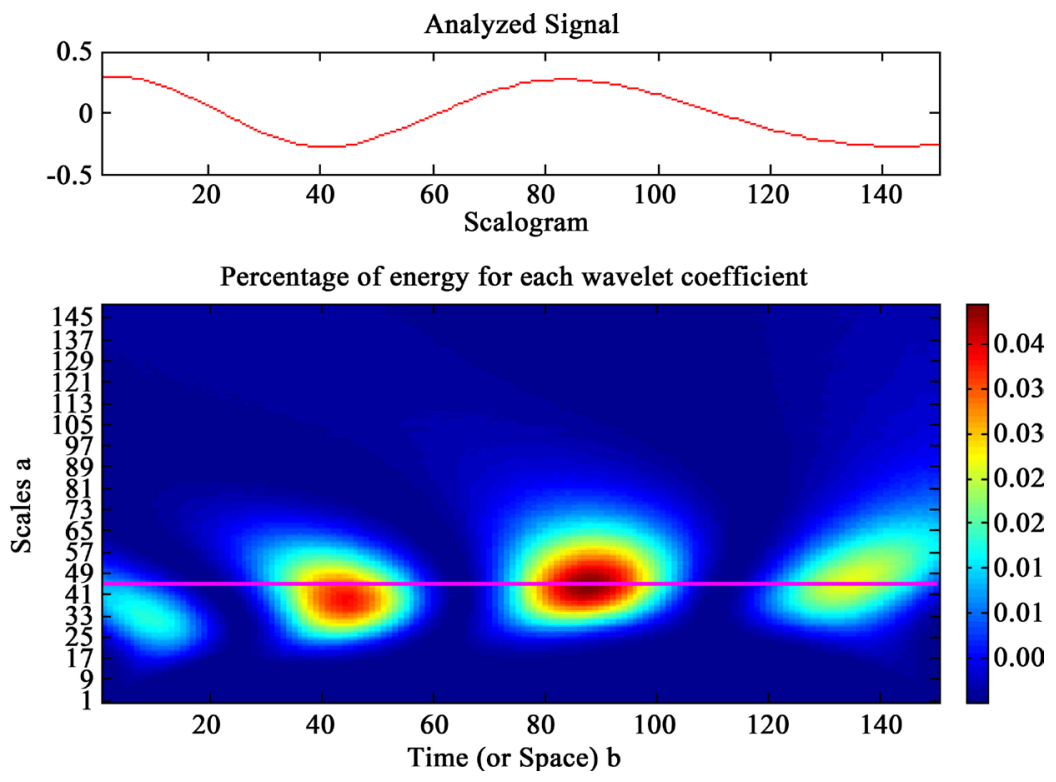

Figure 10. Scalogram of fourth IMF obtained from wavelet transform.

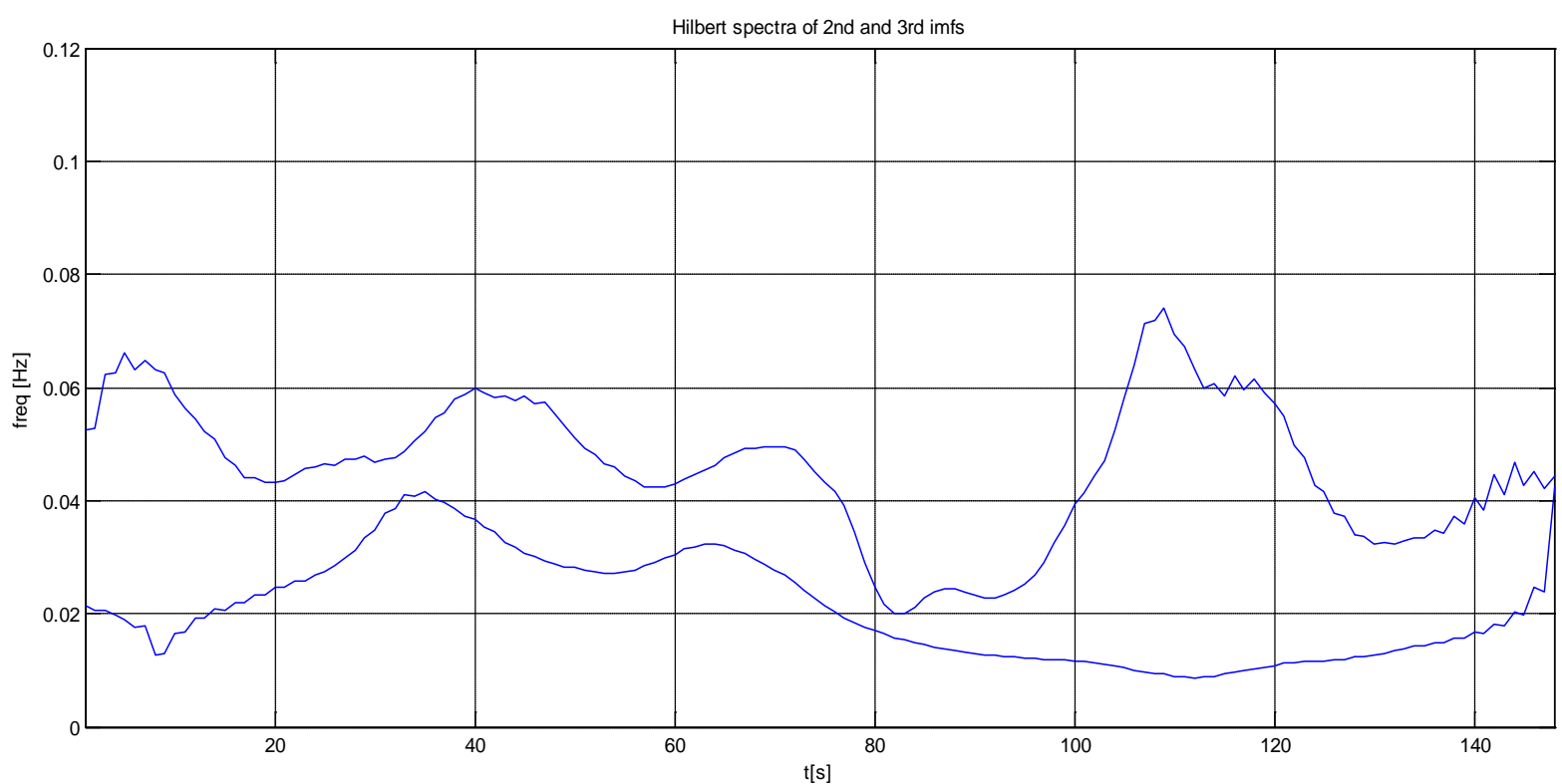

Figure 11. Instantaneous frequency obtained from HHT. 
Table 1. Comparison of different methods of signal analysis.

\begin{tabular}{cccc}
\hline Method & HHT & Fourier & Wavelet \\
\hline Basis & Adaptive & Non-adaptive & Non-adaptive \\
Frequency & Differentiation: Local & Convolution: Global & Convolution: Regional \\
Variable presentation & Energy-time-frequency & Energy-Frequency & Energy-time-frequency \\
Non-linear analysis & Yes & NO & No \\
Non-stationary analysis & Yes & No & Yes \\
\hline
\end{tabular}

quency waves as opposed to single frequency obtained using simple Hilbert Transform (SHT), which only computes the low frequency as given by HHT. The analyses confirm that the original data is a superposition of two waves. The turbulent nature of the magnetosheath magnetic field [17]-[20] indicates the possibility of this superposition of wave from the magnetosphere and the solar wind plasma wave. This analysis has also shown that space plasma waves can be analysed using HHT. The result of this study has shown that (HHT) provides the best applicability in the magnetosheath data analysis than the wavelet and Fast Fourier Transform (FFT). The derivable advantages of HHT in comparison to other methods is viewed to be very useful in the analysis of multi-frequency signal where different frequencies could be determined distinctively at point.

\section{Acknowledgements}

The authors are grateful to Akwa Ibom State University that provided the needed resources, which made this study successful. The editor and the anonymous reviewers are highly acknowledged for their critical comments and suggestions which have improved the quality of the original manuscript.

\section{References}

[1] Nathaniel, E.U., Bellof, N. and George, N.J. (2013) Instantaneous Frequency and Wave Mode Identification in a Magnetosheath Using Few Spatial Points. Chinese Physics B, 22, Article ID: 084701. http://dx.doi.org/10.1088/1674-1056/22/8/084701

[2] Huang, N.E., Tung, C.C. and Long, S.R. (1990) Wave Spectra. The Sea, International Conference of Computational Harmonic Analysis (Invited Talk), 9, 197-237.

[3] Li, H.L., Deng, X.Y. and Dai, H.L. (2007) Structural Damage Detection Using the Combination Method of EMD and Wavelet Analysis. Mechanical Systems and Signal Processing, 21, 298-306. http://dx.doi.org/10.1016/j.ymssp.2006.05.001

[4] Li, H., Deng, X. and Dai, H. (2007) Structural Damage Detection Using the Combination Method of EMD and Wavelet Analysis. Mechanical Systems and Signal Processing, 21, 298-306. http://dx.doi.org/10.1016/j.ymssp.2006.05.001

[5] Nathaniel, E.U., George, N.J. and Etul, S.E. (2013) Determination of Instantaneous Frequencies of Low Plasma Waves in the Magnetosheath Using Empirical Mode Decomposition (EMD) and Hilbert Transform (HT). Atmospheric and Climate Sciences, 3, 576-580. http://dx.doi.org/10.4236/acs.2013.34060

[6] Gabor, D. (1946) Theory of Communication. Journal of Institute of Electrical and Electronic Engineers, 93, 429-457.

[7] Cohen, L. (1989) Time-Frequency Distributions. A Review. Proceedings of the IEEE, 77, 941-981. http://dx.doi.org/10.1109/5.30749

[8] Cohen, L. (1995) Time-Frequency Analysis. Prentice-Hall.

[9] Boashash, B. (1992) Estimating and Interpreting the Instantaneous Frequency of a Signal—Part 1: Fundamentals. Proceedings of the IEEE, $\mathbf{8 0 .}$

[10] Berkant, T. and Loughlin, P.J. (1995) Instantaneous Frequency and Time-Distributions. Proceedings of the IEEE, 10131016.

[11] Flandrin, P., Rilling, G. and Goncalves, P. (2004) Empirical Mode Decomposition as a Filter Bank. Signal Processing Letters, 11, 112-114. http://dx.doi.org/10.1109/LSP.2003.821662

[12] Paschmann, G. and Daly, P. (1998) Analysis Methods for Multi-Spacecraft Data. Scientific Report SR-001, ESA Publications Division.

[13] Denton, R.E., Gary, S.P., Li, X., Anderson, B.J., Labelle, J.W. and Lessard, M. (1995) Low-Frequency Fluctuations in the Magnetosheath near the Magnetopause. Journal of Geophysical Research, 100, 5665-5679. 
http://dx.doi.org/10.1029/94JA03024

[14] Song, P., Russell, C.T. and Gary, S.P. (1994) Identification of Low Frequency Fluctuations in the Terrestrial Magnetosheath. Journal of Geophysical Research, 99, 6011-6025. http://dx.doi.org/10.1029/93JA03300

[15] Shevyrev, N.N., Zastenker, G.N., Nozdrachev, M.N., Nèmećek, Z., Šafránková, J. and Richardson, J.D. (2003) High and Low Frequency Large Amplitude Variations of Plasma and Magnetic Field in the Magnetosheath: Radial Profile and Some Features. Advances in Space Research, 31, 1389-1394.

[16] Carozzi, T.D., Buckley, A.M. and Gough, M.P. (2004) Instantaneous Wave Vector Estimation from Multi-Spacecraft Measurements Using Few Spatial Points. Annales Geophysicae, 22, 2633-2641. http://dx.doi.org/10.5194/angeo-22-2633-2004

[17] Hubert, D. (1994) Nature and Origin of Wave Modes in the Dayside Earth Magnetosheath. Advances in Space Research, 14, 55-64. http://dx.doi.org/10.1016/0273-1177(94)90048-5

[18] Huang, N.E., Shen, Z., Long, S.R., Wu, M.C., Shih, H.H., Zheng, Q., Yen, N., Tung, C.C. and Liu, H.H. (1998) The Empirical Mode Decomposition and the Hilbert Spectrum for Non-Linear and Non-Stationary Time Series Analysis. Proceedings of the Royal Society of London A, 454, 903-995. http://dx.doi.org/10.1098/rspa.1998.0193

[19] Patrick, F., Rilling, G. and Goncalves, P. (2004) Empirical Mode Decomposition as a Filter Bank. IEEE Signal Processing Letters, 11, 112-114. http://dx.doi.org/10.1109/LSP.2003.821662

[20] Constantinescu, O.D., Glassmeier, K.H., Treumann, R. and Fornacon, K.H. (2003) Magnetic Mirror Structures Observed by Cluster in the Magnetosheath. Geophysical Research Letters, 30. http://dx.doi.org/10.1029/2003GL017313 